\title{
OPTIMIZED WIGGLER MAGNET FOR CESR
}

\author{
A. Mikhailichenko, Cornell University, Wilson Lab., Ithaca, NY 14853
}

\section{Abstract}

For CESR operation around $\psi$, Charm and Tau thresholds, the superconducting wigglers with $2.1 \mathrm{~T}$ peak field of $\sim 20 \mathrm{~m}$ total length will be installed around the ring, allowing necessary damping. We describe here optimization process for the wiggler magnet parameters to meet the field value and quality required

\section{INTRODUCTION}

Development of this subject was initiated by proposal considered to modernize the CESR for the low energy operation [1]. Mostly results and procedures developed can be applied to any wiggler however.

It is known [2], that a passage with an angle through the edge field of a multipole, acting to the particle as the next order multipole. This can be expressed as the following

$$
\int_{\text {outside }}^{\text {insude }} G_{m}(s) d s=\frac{\tan \alpha}{m} G_{m-1}(\text { in }) .
$$

Here $G_{m-1}(i n)$ is the value of original multipole at the center, and $G_{m}(s)$ is treated as effective next order multipole as a function of longitudinal distance $s, \alpha$ is a crossing angle. Dipole corresponds to $m=1, G_{0} \equiv B$, quadrupole to $m=2 \ldots$ Mostly used edge focusing in a dipole field is a lowest among this kind of relation. In a wiggler the effective angle is significantly changes during the passage trough the edge, Fig.1. Each wiggler with sinusoidal dependence of the field on longitudinal coordinate $s, B(s)=B_{0} \operatorname{Sin}(s / \lambda)$, where $\lambda=2 \pi \lambda$ is a wiggler period, $B_{0}$ stands for the field amplitude, can be characterized by the dimensionless wiggler parameter $K$, what is $K=e B_{0} \lambda / m c^{2} \cong 93.4 \times B_{0}[T] \times \lambda[m]$.

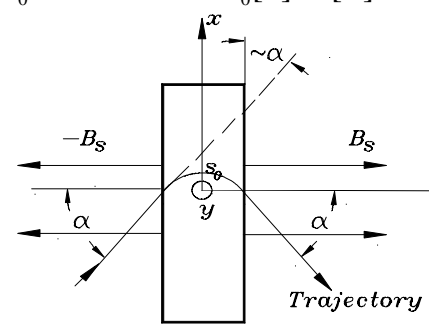

Figure 1: The top view onto the pole of the wiggler. The $y$ - axis is looking up from the plane of the drawing. Dashed line describes the trajectory in a low bend magnet, say quadrupole.

The particle's trajectory in infinite sin-like field with amplitude $B_{0}$ could be described as

$$
x=X+x_{0} \operatorname{Cos}(s / \lambda), \quad \alpha=\alpha_{i n}+\alpha_{0} \operatorname{Sin}(s / \lambda),
$$

where $x_{0}=\lambda K / \gamma, \alpha_{0}=K / \gamma, c$ is a speed of light, $\beta_{\perp 0} \cong K / \gamma, X$ is a constant which depends on details of the entrance in the wiggler field such as tapering. For example, if the wiggler tapered with $+\frac{1}{4},-\frac{3}{4},+1,-1, \ldots$, the constant $X \cong 0$ [4]. The physical nature of appearance of octupole (and higher) type dependence of kick versus displacement is in wiggling with angle in sextupole (and higher) field. This sextupole generated intrinsically by the field dependence on longitudinal coordinate (along the wiggler). When the pole is wide, the sextupole has strict value defined by second derivative of the wiggler field along the longitudinal coordinate $s$.

\section{FIELD IN A WIGGLER}

Static magnetic field existing in a wiggler can be represented as the following [3] ( $S$ and $D$ stand for sextupole and decapole respectively)

$$
\begin{aligned}
& B_{x}(x, y, s)=-\frac{x y}{4} B^{\prime \prime}(s)+\frac{x^{3} y+x y^{3}}{48} B^{(I V)}(s)-\cdots \\
& +2 S(s) x y-\frac{3 x^{3} y+x y^{3}}{12} S^{\prime \prime}(s)+\frac{3 x^{5} y+4 x^{3} \cdot y^{3}+x y^{5}}{32 \cdot 10} S^{(I V)}(\mathrm{s})-\cdots \\
& +4 D(s) x y\left(x^{2}-y^{2}\right)-\frac{15 x^{5} y-10 x^{3} y^{3}-9 x y^{5}}{32 \cdot 7 \cdot 15} D^{\prime \prime}(s)+\cdots \\
& B_{y}=B(s)-\frac{x^{2}+3 y^{2}}{8} B^{\prime \prime}(s)+\frac{x^{4}+6 x^{2} y^{2}+5 y^{4}}{32 \cdot 6} B^{(I V)}(s)-\cdots \\
& +S(s)\left(x^{2}-y^{2}\right)-\frac{3 x^{4}+6 x^{2} y^{2}-5 y^{4}}{12 \cdot 4} S^{\prime \prime}(s)+ \\
& \frac{+3 x^{6}+17 x^{4} y^{2}+5 x^{2} y^{4}-7 y^{6}}{32 \cdot 60} S^{(I V)}(s)-\cdots \\
& +D(s)\left(x^{4}+y^{4}-6 x^{2} y^{2}\right)-\frac{5 x^{6}-15 x^{4} y^{2}-45 x^{2} y^{4}+7 y^{6}}{32 \cdot 7 \cdot 30} D^{\prime \prime}(s)+\cdots \\
& B_{s}=y \cdot B^{\prime}(s)-\frac{x^{2} y+y^{3}}{8} B^{\prime \prime \prime}(s)+\frac{\left(x^{2}+y^{2}\right)^{2} y}{32 \cdot 6} B^{(V)}(s)-\cdots \\
& +\left(S^{\prime}(s)-\frac{x^{2}+y^{2}}{16} S^{\prime \prime \prime}(s)+\frac{\left(x^{2}+y^{2}\right)^{2}}{32 \cdot 20} S^{(V)}(s)-\cdots\right) \frac{3 x^{2} y-y^{3}}{3} \\
& +\left(D^{\prime}(s)-\frac{x^{2}+y^{2}}{24} D^{\prime \prime \prime}(s)+\frac{\left(x^{2}+y^{2}\right)^{2}}{32 \cdot 42} D^{(v)}(s)-\cdots\right) \frac{5 x^{4} y-10 x^{2} y^{3}+y^{5}}{5}
\end{aligned}
$$

For the wiggler with infinitely wide poles in $x$ (transverse) direction, there must be no dependence on $x$, however. This yields the following relations between multipoles

$$
S(s)=\frac{1}{8} B^{\prime \prime}(s), D(s)=\frac{1}{16} S^{\prime \prime}(s)-\frac{1}{192} B^{(I V)}(s), \ldots .
$$

Under these conditions all components of magnetic field from different multipoles along transverse axis $x$ equates to zero, $B_{x}(s)=0$. If we suggest in addition, that the dipole field have sinusoidal dependence on longitudinal coordinate $B(s)=B_{0} \operatorname{Sin}(s / \lambda)$, the multipoles become

$$
S(s)=-\frac{B_{0}}{8 \pi^{2}} \operatorname{Sin}(s / \lambda), \quad D(s) \cong \frac{B_{0}}{384 \cdot \pi^{4}} \operatorname{Sin}(s / \lambda), \ldots
$$

Substitute these values into expression for the fields, one can find, that the field dependence becomes 


$$
\begin{gathered}
B_{x}(y, s)=0, B_{y}(y=0, s)=\sum B_{y}^{m}=B_{0} \cdot \operatorname{Sin}(s / \lambda), \\
B_{s}(y, s) \cong(y / \lambda) \cdot B_{0} \operatorname{Cos}(s / \hbar)-\frac{5}{24}(y / \lambda)^{3} \cdot \operatorname{Cos}(s / \lambda)- \\
-\frac{29}{5 \cdot 384} \cdot(y / \lambda)^{5} \cdot B_{0} \operatorname{Cos}(s / \lambda)+\ldots
\end{gathered}
$$

First term here responsible for linear, the second one for octupole and the last one for twelve pole type focusing along vertical direction. So one can see, that cancellation of the field dependence along one coordinate, $x$, makes dependence along other one, $y$, more stiff.

Vertical force acting to the particle with instant position $(x, y, s)$ is proportional to the

$$
F_{y} \cong e \vec{v} \times\left(-B_{s}(x, y, s) \cdot \operatorname{Sin} \alpha(x, y, s)+B_{x}(x, y, s)\right),
$$

where $\vec{v}$ is the particle's speed. Vertical kick experienced by a particle, passed though the multipole magnet off its center with angle $\alpha$, can be expressed as the following

$$
y^{\prime} \cong \frac{1}{p c} \int_{\text {outside }}^{\text {inside }} \frac{F_{y}(y, s) d s}{\cos \alpha} \cong-\frac{1}{B R} \int_{\text {outside }}^{\text {inside }} B_{s}(y, s) \cdot \tan \alpha \cdot d s,
$$

where $e / p c=B R$ is a magnet rigidity. So sin-lke motion brings additional factor $1 / 2$ in this formula (and in formula (1)).

\section{THE OCTUPOLE VALUE}

The tune shift generated by octupole $O(s)$ is [5]

$$
\Delta Q \cong \frac{3}{8} \beta_{0} \cdot a^{2} \cdot N \cdot \int O(s) \cdot d s /(H R),
$$

where $\beta_{0}$ is an envelope function at the wiggler's location, $N$ is the number of wigglers in all machine ( $N=14$ in our case). We suggested for simplicity, that all wigglers (octupoles) be displaced around the ring having the same envelope function and phase. So this is majorette estimation. Meanwhile we could see from previous considerations, that

$$
\int O(s) d s \cong \frac{5}{2 \cdot 24} \frac{\alpha_{0} B_{0}}{\hbar^{2}} \cdot(L / \pi \hbar-1) \times N,
$$

Factor $1 / 2$ reflects sinusoidal motion.

Let us estimate (2) for $\Delta Q \cong 2 / 100$ allowed. For $\beta_{0} \cong 10 \mathrm{~m},(H R) \cong 6666 \mathrm{kG} \mathrm{cm} \quad(2 \mathrm{GeV}), a \cong 2 \mathrm{~cm}$, formula (2) yields $\int O \cdot d s \leq 0.089 \mathrm{kG} \cdot \mathrm{cm}^{2}$. From the other hand, formula (3) for $B_{0} \cong 2 T, \quad \lambda \cong 6.37 \mathrm{~cm}, \quad K \cong 75$, $\alpha \cong 0.019, L=150 \mathrm{~cm}$, yields $\int O(s) d s \cong 0.086 \mathrm{kG} \cdot \mathrm{cm}$. So for this example the shift is about $\Delta Q \cong 0.02$ for the particle having amplitude $a=2 \mathrm{~cm}$.

Let us calculate now the ratio of linear part to the cubic one for the same wiggler set. Integral of linear part for all $N$ wigglers goes to

$$
\int G(s) d s \cong \frac{\alpha_{0} B_{0}}{2} \times(L / \pi \hbar-1) \times N,
$$

where factor $1 / 2$ appeared for sin-like trajectory. So the ratio of octupole to linear terms goes for arbitrary amplitude $a$ to $\delta=a^{2} \int O(s) d s / \int G(s) d s \cong \frac{5}{24}\left(a^{2} / \hbar^{2}\right)$ For our example if $a=2 \mathrm{~cm}$, then $\delta \cong 2 \%$.
With additional real octupole installed in the ring one may try to reduce the influence of nonlinear term. Problem here is that wiggler focusing only in vertical direction. The simplest way to reduce the octupole term is to increase the wiggler period $\lambda$ and/or $B_{0}$, as the dependence is a quadratic one. With increasing the wiggler period, however, at some moment the term with derivative comes to $S \cong \frac{1}{8} \partial^{2} B / \partial s^{2} \rightarrow \frac{1}{8} B_{0} / A^{2}$, where $A$ is a vertical aperture of the wiggler. In this case the octupole integral becomes about two times higher, as the particle passes fringe field with higher angle. From the other hand if the damping time is fixed, its dependence on $B_{0}^{2}$ what gives the factor 2 in extreme case back.

Sinusoidal field dependence and trajectory can be restored either by increasing the vertical gap (with increasing the feeding current) or by changing distribution of the winding, making the coil wider (and flatter).

\section{PROCEDURE}

Formulas represented above give an understanding of analytical dependencies on different parameters. For more realistic optimization the following sequence was used. First, 3D calculations were carried with real dimensions and multi-wired currents with all possible details with the help of numerical code [6].

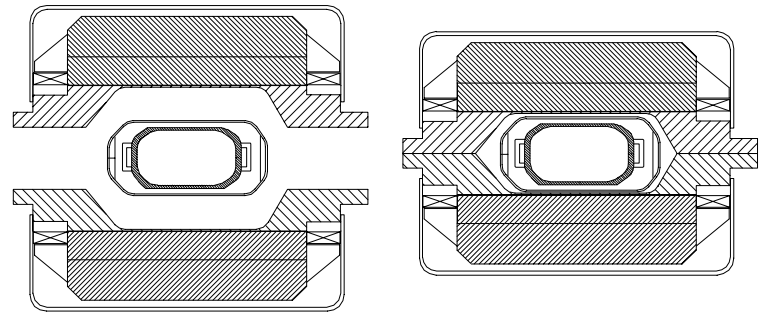

Figure 2: The wiggler's cold mass cross section. Room temperature aperture is $9 \times 5 \mathrm{~cm}^{2}$.

Dense hatch in Fig. 2 related to the soft iron, rare -to Stainless Steel. Iron begins at $1.5 \mathrm{in}$ from the median plane. Upper and lower containers for liquid Helium are connected through the side tubing. This design drastically simplifies assembling, excluding, however, any magnet shunts between upper and lower parts inside a cryostat. During the modeling, the magnet was plunged into a box with appropriate margins. In longitudinal direction the sides of this box coincide with the positions of the cryostat flanges, located at $20-\mathrm{cm}$ distance from the yoke. Magnetic field was investigated everywhere in the box and forces acting to the coils calculated. Optimization of the iron thickness, pole shapes and tapering, shims, field profile above the iron and so on were done here. Energy stored in the field (and inductance) obtained as well. Influence of the stainless steel magnetic permeability was investigated also (Fig.2), coming to restriction on $\mu_{r} \leq$ 1.02. Possible screening of HTS leads with local iron cylinders was evaluated also. 
After the field distribution obtained, the tracking through the box was carried with the help of another numerical code [7]. After post processing, all necessary parameters of the particles represented as a function of transverse phase space location at the entrance of the wiggler.

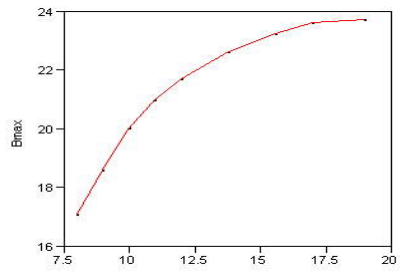

Figure 3: Field in the gap, $k G$, as a function of the iron height, $\mathrm{cm}$, measured from median plane on Fig.2.

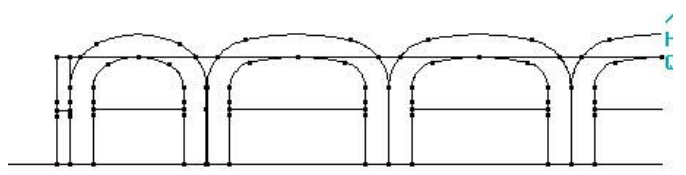

Figure 4: Top view on the wiggler poles.

Half of the wiggler is represented in Fig.4. Regular pole length is $20 \mathrm{~cm}$. The end pole length is $15 \mathrm{~cm}$. Radiuses chosen for easy winding and to lower the local field strength. The smallest one in the Fig. 4 is $2.9 \mathrm{~cm}$. The coil width is $2.54 \mathrm{~cm}$. The end pole contains main coil caring the same current as the central poles and the trimming SC coil with independent power supply. Poles in regular part have lowering of $5 \mathrm{~mm}$ deep, allowing alignment the field distribution across the pole, see Fig.5. End poles have the lowering depth of $3.5 \mathrm{~mm}$ only. The widths of the lowering are about $\pm 6 \mathrm{~cm}$. This allows having the integral $I_{1}=\int B_{z}(s) d s$ variation across the aperture $I_{1} \leq 0.05 \mathrm{kG} \cdot \mathrm{cm}$, while integral along single pole (from zero to zero) is $318.8 \mathrm{kG} \mathrm{cm}$.

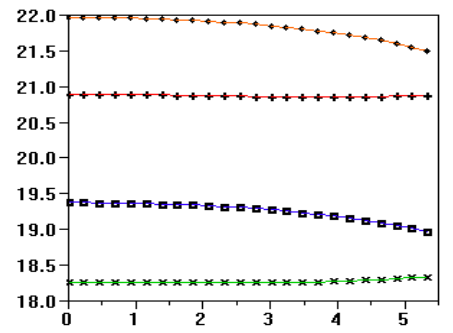

Figure 5: Vertical field, $k G$ as a function of transverse coordinate $x, \mathrm{~cm}$ for two different feeding currents and pole shapes-flat and with lowering. Vacuum chamber ends at $\pm 4.5 \mathrm{~cm}$.

Two upper curves in Fig.5 correspond to the feeding current $100 \mathrm{kA}$ total, flat pole, up, and next lower curve to the modified ones. Two lower curves correspond to feeding current $80 \mathrm{kA}$. Upper among them for flat pole, lower-for the same modified one.

During modeling the steel 1010 properties were used, however we investigated the magnet field variation with other ones, such as 1006, Steel10 for example.
As one can see from Fig.6, oscillations sweep in $x$ direction area about $3.3 \mathrm{~mm}$ wide.
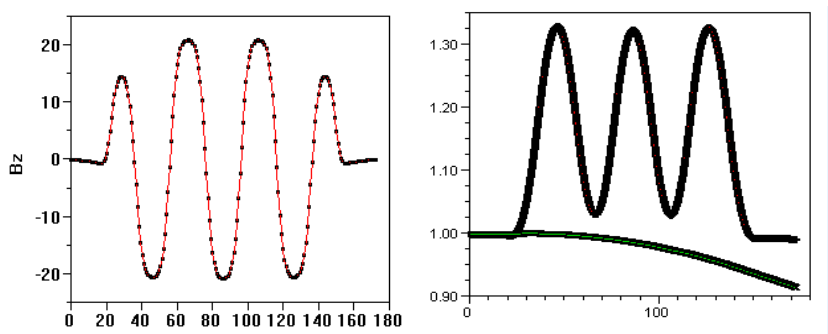

Figure 6: Longitudinal $B_{z}$ profile (left, $k G$ ), and example of particle's trajectory starting at $(x, y)=(1,1)$. Scales at the right are in $\mathrm{cm}$.

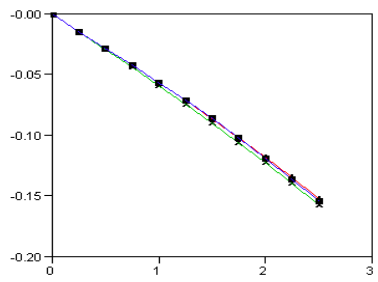

Figure 7: Deviation of vertical position of the particle from initial one as a function of initial vertical position for different horizontal initial $x$-positions.

Parameters of the wiggler are represented in the Table.

\begin{tabular}{|c|c|}
\hline Height of the cold iron & $29 \mathrm{~cm}$ \\
\hline Pole length & $20 / 15 \mathrm{~cm}($ end pole $)$ \\
\hline Number of poles & $5+2$ \\
\hline Current & $150-195 \mathrm{~A}$ \\
\hline Pole width & $23.8 \mathrm{~cm}$ \\
\hline Field & $1.7-2.1 T$ \\
\hline Cubic /linear kick ratio & $\delta \leq 8 \% @ 2 \mathrm{~cm} \mathrm{in}|x| \leq 5 \mathrm{~cm}$ \\
\hline Energy stored & $47.3 \mathrm{~kJ}($ at $2.2 \mathrm{~T})$ \\
\hline
\end{tabular}

Work supported by National Science Foundation.

\section{REFERENCES}

[1] G. Codner et all., Parameters for Low Energy operation of CESR, These proceedings, WOAB011.

[2] A. A. Mikhailichenko, Betatron Tune Shift Generated specifically in the Pretzel Machine, CBN 98-4, February 10, 1998

[3] A.A. Mikhailichenko, 3-D electromagnetic Field. Representation and measurements, CBN 95-16, Cornell, 1995, $42 \mathrm{pp}$.

[4] A. A. Mikhailichenko, V.V. Parkhomchuk, Damping Ring for a Linear Collider, BINP Preprint 91-79, Novosibirsk, 1991.

[5] A. Mikhailichenko, Nonlinear effects in a Wiggler, CBN 01-07, Cornell, 2001.

[6] MERMAID -MEsh oriented Routine for MAgnet Interactive Design, SIM Limited, Novosibirsk, P.O. Box 402, Russia.

[7] G.Dudnikova, V.Vshivkov, K.Vshivkov, UMKA-VG, Institute of Computation Technologies, Lab. of Plasma Physics, Siberian Branch of RAN. 